\title{
Light limitation increases multidimensional trait evenness in phytoplankton populations
}

\author{
Simone Fontana $\mathbb{1}^{1,3} \cdot$ Mridul K. Thomas $\mathbb{B}^{1,4} \cdot$ Marta Reyes $\mathbb{B}^{1} \cdot$ Francesco Pomati $\mathbb{C}^{1,2}$
}

Received: 2 July 2018 / Revised: 10 October 2018 / Accepted: 4 November 2018 / Published online: 7 January 2019

(c) International Society for Microbial Ecology 2019

\begin{abstract}
Individual-level variation arising from responses to environmental gradients influences population and community dynamics. How such responses empirically relate to the mechanisms that govern species coexistence is, however, poorly understood. Previous results from 1

ake phytoplankton communities suggested that the evenness of organismal traits in multiple dimensions increases with resource limitation, possibly due to resource partitioning at the individual level. Here we experimentally tested the emergence of this pattern by growing two phytoplankton species (Pseudokirchneriella subcapitata and Microcystis aeruginosa) under a gradient of light intensity, in monoculture and jointly. Under low light (resource) conditions, the populations diversified into a wide range of phenotypes, which were evenly distributed in multidimensional trait space (defined by four pigment-related trait dimensions), consistent with the observed field pattern. Our interpretation is that under conditions of light limitation, individual phytoplankton cells alter photosynthetic traits to reduce overlap in light acquisition, acquiring unexploited resources and thereby likely maximising individual success. Our results provide prime experimental evidence that resource limitation increases the evenness of conspecific and heterospecific microbial phenotypes along trait axes, advancing our understanding of trait-based coexistence.
\end{abstract}

\section{Introduction}

Many processes that are fundamental to community assembly, such as responses to abiotic gradients, direct species interactions (e.g., predation, parasitism), and

Supplementary information The online version of this article (https:// doi.org/10.1038/s41396-018-0320-9) contains supplementary material, which is available to authorized users.

Simone Fontana

simone.fontana@wsl.ch

1 Department of Aquatic Ecology, Eawag, Swiss Federal Institute of Aquatic Science and Technology, Überlandstrasse 133, 8600 Dübendorf, Switzerland

2 Institute of Integrative Biology, Swiss Federal Institute of Technology (ETH), Universitätstrasse 16, 8092 Zürich, Switzerland

3 Present address: Biodiversity and Conservation Biology, Swiss Federal Institute for Forest, Snow and Landscape Research WSL, Zürcherstrasse 111, 8903 Birmensdorf, Switzerland

4 Present address: Centre for Ocean Life, DTU Aqua, Technical University of Denmark, Kongens Lyngby, Denmark competition (interspecific as well as intraspecific), act at the level of individual organisms at the smallest temporal and spatial scales $[1,2]$. Stabilising mechanisms of coexistence explicitly invoke the relative importance of intraspecific vs. interspecific competition [3]. Recent studies have bolstered the case for the influence of individual-level trait variation on community assembly and species coexistence [4-11].

Individual-level variation in traits is a feature common to all species, including clonal and microbial populations [12]. Limitation and fluctuations in resources are important drivers of intraspecific phenotypic heterogeneity, which favours population fitness [12-14]. For example, $\mathrm{NH}_{4}{ }^{+}$ limitation can trigger an increase in phenotypic heterogeneity in the $\mathrm{N}_{2}$-fixing bacterium Klebsiella oxytoca, which proves beneficial under resource fluctuations [13]. A decrease in availability of resources like phosphorus and light in lakes was also shown to induce greater evenness (i.e., regularity) of phytoplankton cells' traits, possibly to minimise competition for limiting resources [15]. The latter community-wide pattern observed in nature is probably a consequence of phenotypic changes induced by plasticity and/or selection that stabilise coexistence by allowing for resource partitioning among individuals and species. For 


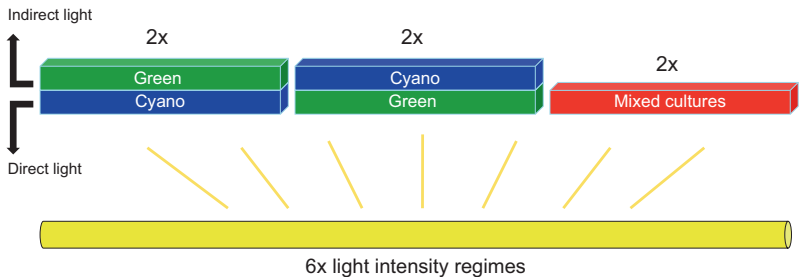

Fig. 1 Illustration of the experimental design. For simplicity, only one out of two replicates and one out of six light treatments are reported. Green $=P$. subcapitata , Blue $=M$. aeruginos $;$ Red $=$ Mixed cultures ( $P$. subcapitata $+M$. aeruginosa) (color figure online)

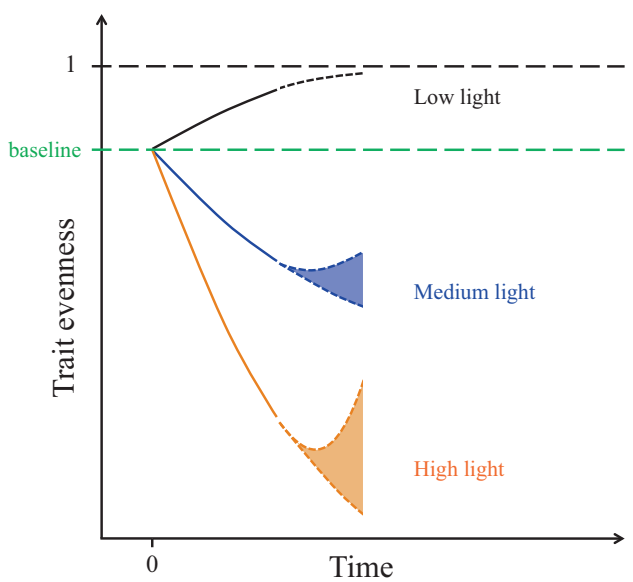

Fig. 2 Expected change of trait evenness over time based on the hypothesis that light limitation and competition induce higher evenness in the photosynthetic traits of individual phytoplankton cells. This arises from the need to absorb wavelengths that have not been used by competitors. The three depicted curves reflect different shifts in the light intensity regime compared to the time before the beginning of the experiment (decrease, moderate increase and strong increase). Dashed black $=$ maximum possible value of trait evenness. Dashed green $=$ baseline value of trait evenness, resulting from the maintenance of all cultures at the same light intensity prior to the experiment. We expect the response to be approximately linear at the beginning, whereas more uncertainty is associated with longer time scales, as indicated by coloured bands and dashed lines (color figure online)

instance, the relative composition and amount of pigments may allow phytoplankton species to partition the light spectrum; different wavelengths can be absorbed in different proportions depending on pigmentation [16, 17].

In this study, we experimentally tested the hypothesis that limitation in resource supply, specifically light availability, increases phenotypic heterogeneity (i.e., the evenness of the photosynthetic trait distribution measured by scanning flow-cytometry) among individual cells. Under competition for light, individual organisms should adjust pigment composition to capture different wavelengths of the light spectrum and reduce niche overlap. This has been observed at the species level [17], and should emerge from both intra- and interspecific competition. Here we exposed two phytoplankton species belonging to different functional groups (the green alga Pseudokirchneriella subcapitata and the cyanobacterium Microcystis aeruginosa) to six different light intensities (Fig. 1), and quantified cell density and trait evenness over time and after reaching carrying capacity. The two species utilise the light spectrum in different ways: both possess chlorophyll (which captures light in the blue and red portions of the visible spectrum), while M. aeruginosa also possesses the accessory pigment phycocyanin (which captures light in the green-yellow portion, for the most part inaccessible to $P$. subcapitata) [18]. Monocultures were tested under direct light and under light passing through a culture of the competing species (hereafter indirect light). In this way, we simulated the effects of competition for light intensity and different parts of the spectrum, while excluding direct interspecific interactions (such as through allelopathy). Additionally, we also tested a mixture of the two species (under direct light only) to account for direct interactions between cells.

If competitive interactions between individual organisms are the driving force of even spacing in acclimating phenotypes, we expect that the rate of change in trait evenness will range from positive to increasingly negative values as light intensity increases (Fig. 2). Under high levels of light, organisms should be freely distributed in multidimensional trait space or shift towards a few optimal trait combinations, leading to a decrease in trait evenness over time. Trait evenness has a maximum possible value (Fig. 2, dotted black line tending to a horizontal asymptote) and should reach a minimum as cell densities eventually induce resource limitation and competition, and therefore an increase in trait evenness (Fig. 2, blue and orange bands indicating a possible reversal of the curves). Our experiment examined the initial response, which can be approximated by a straight line (Fig. 2). Trait evenness values at carrying capacity should also be inversely proportional to light intensity.

\section{Materials and methods}

\section{Experimental conditions and culture acclimation}

In a climate-controlled room with constant temperature of $20{ }^{\circ} \mathrm{C}$, we set up six different light regimes. Osram T8 L De Luxe $36 \mathrm{~W} 954$ G13 Lumilux lights were placed under Plexiglas supports of varying height. The support elevation and several meshes (black and white) were used to vary light intensity (more details on experimental setup in Supplementary Table S1). The ranges of the resulting light intensities (in $\mu \mathrm{E} \mathrm{m}^{-2} \mathrm{~s}^{-1}$ ) at the culture bottles over multiple measurements are presented here: $380-430$ (midpoint $=405), 173-290$ (231.5), 95-155 (125), 60-90 (75), 25-40 (32.5), 0.4-3 (1.7). All treatments followed a $14: 10 \mathrm{~h}$ lightdark cycle. 
P. subcapitata strain SAG 12.81 (from the SAG Culture Collection of Algae, University of Göttingen, Germany) and M. aeruginosa strain PCC 7806 (toxic wild type obtained from Brett A. Neilan, UNSW, Sydney, Australia) were maintained as batch cultures in a separate culturing room under identical environmental conditions. Cultures were maintained in exponential growth phase at $20^{\circ} \mathrm{C}$ and a light intensity of approximately $20-25 \mu \mathrm{E} \mathrm{m}^{-2} \mathrm{~s}^{-1}$ (intermediate between the two lowest light intensity treatments). The initial trait evenness of our test cultures was therefore supposed to be similar at the beginning of the experiment (Fig. 2, dashed green baseline). Replicated cultures were transferred to the different experimental light regimes 10 days before starting the experiment.

We diluted with WC-medium the content of the Erlenmeyer flasks (batch cultures) into cell culture bottles (Faust Lab Science, product number TPP90301) closed with filter caps that allow gas transfer. We applied a 1:20 dilution of the original cultures for all but the two lowest light intensities (1:10 in this case, as we expected a much slower growth under low light regimes), with mixed cultures having equal volumes of the two species, and obtained a final volume of $200 \mathrm{ml}$ in each cell culture bottle.

\section{Experimental design}

We obtained a total of six units in every light treatment compartment: cultures placed on top of each other $(P$. subcapitata above-and shaded by-M. aeruginosa, plus $M$. aeruginosa above $P$. subcapitata, two replicates each) and mixed cultures (two replicates) (Fig. 1). In this way, monocultures of $P$. subcapitata and $M$. aeruginosa were subjected to both direct and indirect light (cell culture bottles were narrow and so did not totally block incoming light), while mixed cultures always experienced direct light. The position of these units within the light treatment compartments was randomised and shuffled regularly. The experimental design is illustrated in Fig. 1.

\section{Logistic growth and carrying capacity}

Cell counts from scanning flow-cytometry were used to derive cell density of each sample. We fitted a logistic growth curve to cell density data from each of the replicates using the function gcFitModel (grofit package, [19]) to obtain carrying capacity estimates (details in Supplementary Table S2). gcFitModel fits several parametric models to growth data, and automatically select the most parsimonious one. Here are the equations of the models in our study:

Logistic function : $f(t)=A /[1+\exp ((4 \mu / A)(\lambda-t)+2)]$,
Gompertz function : $f(t)=A \cdot \exp [-\exp ((\mu \exp (1) / A)(\lambda-t)+1)]$,

Richards function : $f(t)=$

$A \cdot\left[1+\nu \cdot \exp \left(1+\nu+(\mu / A) \cdot(1+\nu)^{(1+1 / \nu)}(\lambda-t)\right)\right]^{(-1 / \nu)}$,

where $A$ is the carrying capacity, $\mu$ is the maximum slope, $\lambda$ is the lag-phase, $\nu$ is the degree of asymmetry, and $t$ is time.

$R^{2}$ values, calculated regressing observed against fitted values, ranged from 0.845 and 0.996 (Supplementary Table S2). In each replicated growth curve (Supplementary Fig. S1-S6) we identified the first time point with density equal to or greater than the carrying capacity estimate. We defined all time points after reaching carrying capacity as the period of maximum competition for resources among individuals, which allowed a meaningful comparison of trait evenness across treatments, independent of the different temporal trajectories, by controlling for the effect of population-specific growth over time.

\section{Culture maintenance and sampling protocol}

Each culture was manually shaken once a day from Monday to Friday to avoid the formation of a biofilm on the flasks' bottom. The three highest light intensity treatments (405, 231.5 and $125 \mu \mathrm{E} \mathrm{m}^{-2} \mathrm{~s}^{-1}$ ) were sampled 10 times between the 9th of March (start of the experiment) and the 1st of April 2016 (every 2-3 days for 24 days), when they had already reached carrying capacity and showed an accumulation of dead cells (based on colour change). The three lowest light treatments $\left(75,32.5\right.$ and $\left.1.7 \mu \mathrm{E} \mathrm{m}^{-2} \mathrm{~s}^{-1}\right)$ were additionally sampled on the 4th, 6th, and 14th of April, and the very lowest also on the 29th of April and on the 11th of May. These additional sampling dates allowed all treatments but the lowest to reach carrying capacity (as defined in the paragraph "Logistic growth and carrying capacity"). At each sampling date we collected $1.5 \mathrm{ml}$ from all cultures in Eppendorf tubes and fixed it with $0.01 \%$ paraformaldehyde and $0.1 \%$ glutaraldehyde for scanning flow-cytometry analyses.

\section{Scanning flow-cytometry measurements}

We characterised the fluorescence profile (related to light acquisition strategy) of individual cells in each population/ community using scanning flow-cytometry. The instrument we used (www.cytobuoy.com, Woerden, The Netherlands) is able to capture scattering and pigment fluorescence of algal cells in a time resolved mode (scanning) [20-22]. The instrument is specifically designed for high resolution scanning of freshwater phytoplankton pigment fluorescence emission. The scanning flow-cytometer was equipped with 
two laser beams (coherent solid-state sapphire) with excitation wavelengths of $488 \mathrm{~nm}$ (blue light) and $635 \mathrm{~nm}$ (red light), which can excite both the primary and some accessory algal pigments. Four detectors captured emitted pigment fluorescence in the red $(677-700 \mathrm{~nm}$, mainly chlorophyll- $a)$, orange $(650-677 \mathrm{~nm}$, for phycocyanin), yellow (590-620 nm, for phycoerythrin), and green (550-570 nm, for carotenoids) ranges. Data acquisition during this study was triggered by sideward scattering (SWS signal, trigger threshold $30 \mathrm{mV}$ ), and the flow speed was set to $1.05 \mu \mathrm{l} \mathrm{s}^{-1}$. We measured approximately between 6000 and 99,000 cells depending on cell densities.

Applying a clustering algorithm (flowPeaks package, [19]) to a subset of 100,000 particles built by randomly sampling an equal number of particles from all experimental samples, we were able to define distinct clusters of organic debris/suspended solids (characterized by low FL), P. subcapitata and $M$. aeruginosa (the second having a lower red to orange fluorescence ratio). Using this sample dataset, we trained a random forest classifying algorithm [23, 24] and assigned every particle in the whole dataset to one of the three abovementioned categories. The data cleaning and clustering procedure is described in detail in Thomas et al. [25].

\section{Individual-based trait distribution}

The regularity in the distribution of individual cells in multidimensional trait space was quantified for all samples using the TED index ('trait even distribution'; [26]), which compares the probability density function (kernel density estimation) of pairwise distances between individuals in a target population or community and in a perfectly even reference trait distribution. For the calculation of this multivariate index four traits derived from scanning flow-cyometry were used, which should reflect ability to compete for light: total fluorescence in each of the red, orange, yellow and green channels. These traits are associated with pigment quantity and activity, and are therefore indicators of relative investment of individual cells in capturing different portions of the light spectrum $[15,21,27]$. A subset of 5000 randomly selected cells was used to calculate TED. For mixed cultures, we additionally calculated species-specific TED indices with a random subset of 1000 cells for each species. To better understand and interpret complex changes in multidimensional trait distributions, we also calculated the TOP index of trait richness ('trait onion peeling'; [26]) using the same subsets of cells used for TED. TOP is a measure that takes into account all individual phenotypes (including intermediate ones), and thus does not simply represent a multidimensional range but rather an estimation of the trait space effectively covered by individuals [26]. The area of the convex hull containing all phenotypes in multidimensional trait space is determined, and the successive area is calculated upon removal of the previous convex hull's vertices. This procedure continues until all phenotypes are included, and the sum of all areas obtained is the TOP index. A visual explanation of TED and TOP index is provided in Supplementary Fig. S7.

\section{Statistical analyses}

Our experiment covered a time interval insufficient to detect clear curvilinear trends, and we expected the response to be approximately linear at the beginning (Fig. 2). Therefore, we used simple linear regressions as the most parsimonious common approach for all analyses (after checking model assumptions visually).

To test whether trait evenness represented a response to light limitation, we performed two different analyses considering $P$. subcapitata and $M$. aeruginosa separately. First, we fitted linear regressions for each treatment combination (of light intensity and shading) separately (Fig. 3), with date of sampling as predictor and TED as response variable. All slope estimates were then included as response variable in linear models, using least squares. Points were weighted by the inverse of their standard errors (to account for uncertainty in the slope estimates) and light intensity midpoint (mean value of minimum and maximum measured for each treatment) used as predictor. Second, using the time points at carrying capacity detected in replicates (as defined in the paragraph "Logistic growth and carrying capacity"), we fitted linear models to test the relationship between light intensity midpoint (predictor) and TED at carrying capacity. Replicates of the lowest light treatment did not reach carrying capacity and consequently were omitted from these linear models. This analysis aimed at testing the influence of light intensity on TED by correcting for its temporal change, which varied among different cultures. Analysis of covariance (ANCOVA) was used to test whether competition treatments ("direct light", "indirect light" and "mixed cultures") influenced the effect of light intensity on the rate of change of TED and TED at carrying capacity in the two above-mentioned analyses. To this end, using the function aov [19] we included the interaction between shading and light intensity in the global models. We calculated type-III analysis-of-variance tables (orthogonal contrasts setting) with the function Anova (car package, [19]) to perform $F$-tests on the explanatory variables.

\section{Results}

All results were consistent with the expectation that decreasing light intensity induces an increase in the evenness of individual phytoplankton in multidimensional photosynthetic trait space. 


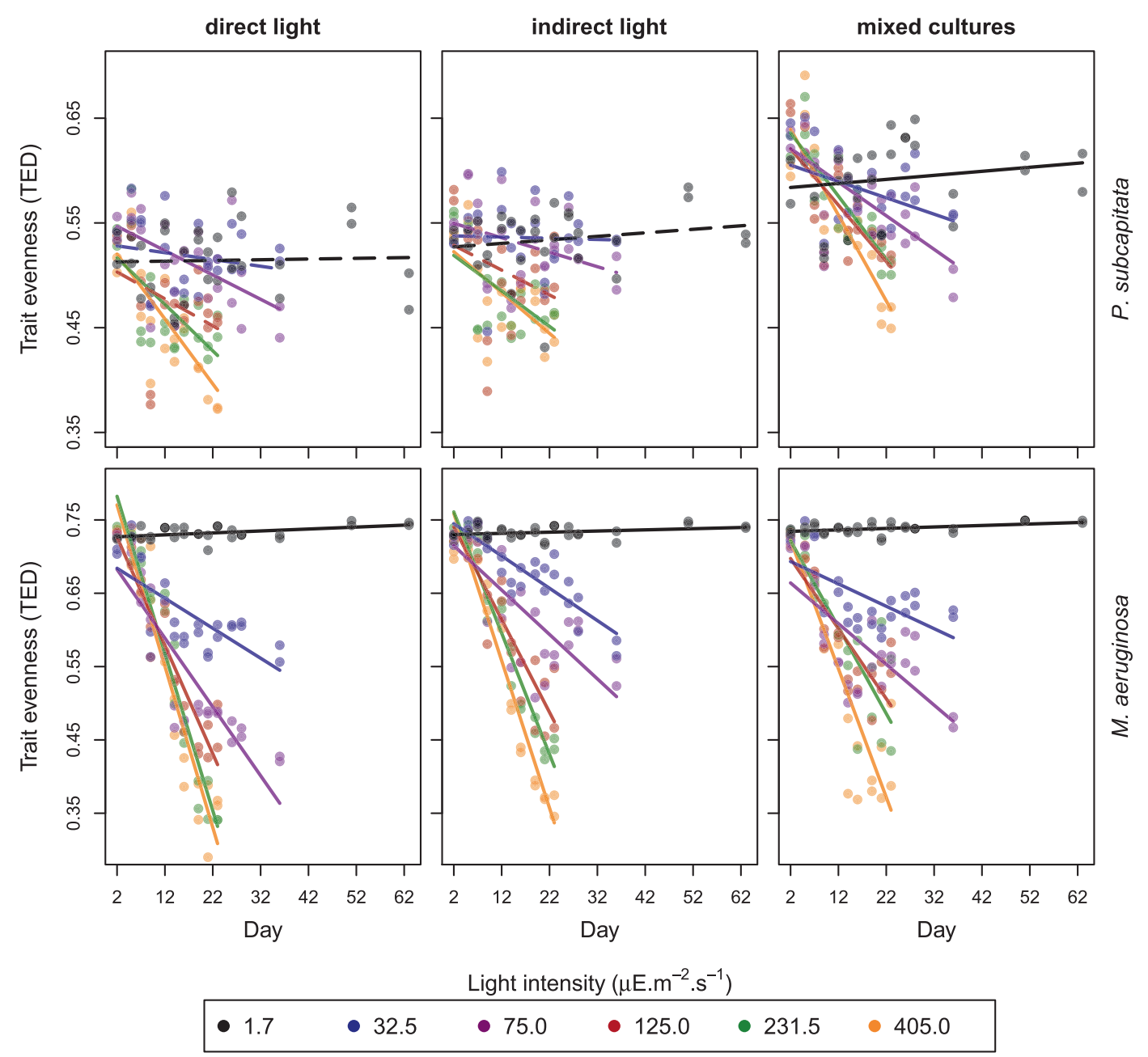

Fig. 3 Change in trait evenness (TED index) over time. Lines represent independent linear model fits at each light level for each species (that we were able to distinguish also in mixed cultures, see Methods section) and in each shading category. Dashed lines indicate non- significant relationships. Note that higher light intensity treatments stop earlier, as they reach carrying capacity faster and consequently show an accumulation of dead cells (color figure online)

competition treatments (including mixed cultures) did not differ significantly, as indicated by the addition of the interaction between light intensity and shading in the global model (P. subcapitata: $p=0.10 ; M$. aeruginosa: $p=0.41$ ).

Trait evenness at carrying capacity also decreased with increasing light intensity (Fig. 4, bottom panels); this decrease was statistically significant in all linear models with the exception of $P$. subcapitata in mixed cultures (Table 1b). Explained variance ranged between 12 and $81 \%$ (Table 1b). The slopes of the three competition treatments were not significantly different for $M$. aeruginosa (interaction between light intensity and shading: $p=0.10$ ). On the contrary, in $P$. subcapitata, trait evenness at carrying capacity showed a more negative slope under direct than indirect light (Fig. 4, bottom panels), as shown by the significant interaction $(p=0.01)$ between light intensity and shading in the global model. 

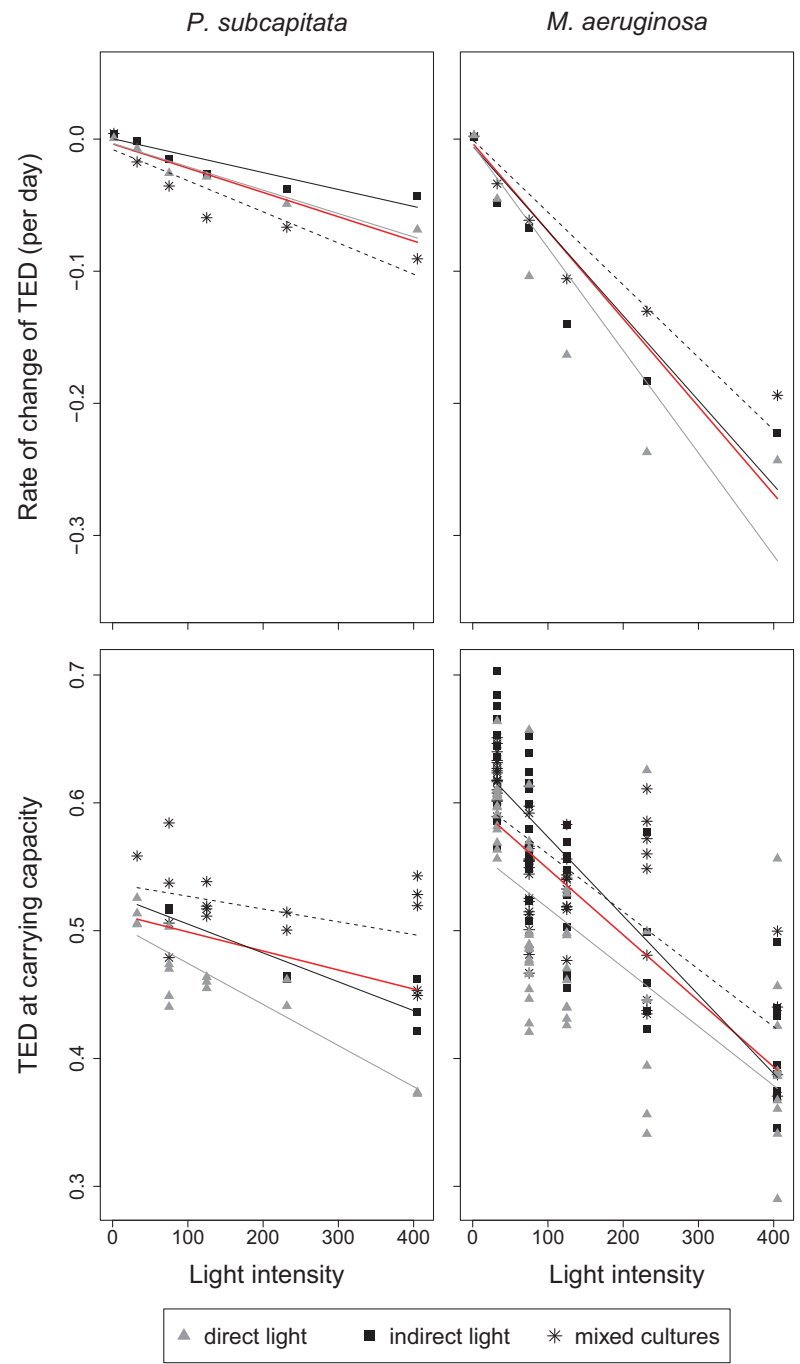

Fig. 4 Relationship between light intensity and two response variables: the rate of change in TED with time i.e., the slope of the regression between TED and time (top panels), and TED values at carrying capacity (bottom panels). Linear fit lines are indicated (dashed = mixed cultures, red = global fit). More complex models (e.g. exponential decay) did not improve the goodness of fit significantly and would not affect our conclusions. Note that in the bottom panel the number of points at each light intensity is not constant, because for each replicate we only included samples collected after reaching the carrying capacity (e.g., no samples in the lowest light treatment; Supplementary Fig. S1-S6) (color figure online)

\section{Discussion}

Our data show strong experimental evidence that (1) variation in resource availability leads to changes in phenotypic trait combinations in phytoplankton that are detectable at both interspecific and intraspecific levels and, (2) under limited resource (light) supply, trait similarity among cells tends to be minimised. Phytoplankton increased their phenotypic heterogeneity concomitantly reducing their trait similarity with heterospecific or conspecific individuals. We interpret this to mean that under conditions of light limitation, individual phytoplankton cells alter their phenotype in a manner that reduces overlap in photosynthetic traits, thereby increasing light acquisition and maximising individual success. This leads to an increase in trait evenness when light intensity is low.

It has been suggested that considering several components of trait diversity can help characterise complex changes in multidimensional trait space and effectively link phenotypic variation to community structural and functional properties [15, 26, 28]. In this study, we used different light regimes to manipulate resource limitation and competition, although one could argue that light limitation represents an environmental factor that typically constrains the number of viable trait combinations, thus reducing trait range and consequently increasing phenotypic similarity [29, 30]. Our observed patterns of an increase in trait evenness are not in contradiction with a possible, simultaneous contraction of the trait space covered (which would constitute a reduction in trait richness). We note here that TED, our metric of trait evenness, measures trait regularity independent of the absolute distances between individual phenotypes [26] and reflects the tendency to maximise those distances given any trait range. Thus, trait evenness is not necessarily related to the overall trait space covered (i.e., trait richness). In our data, trait richness (TOP index) showed a pattern very similar to trait evenness (TED) in M. aeruginosa (Supplementary Fig. S8). In contrast, trait richness in P. subcapitata did not change in a consistent way over time and across light intensity treatments (Supplementary Fig. S8). In other words, M. aeruginosa increased both trait evenness and richness as a response to low light, whereas $P$. subcapitata mainly maximised evenness within the available and constant trait space. This result was probably due to a lower plasticity in pigment-related traits in $P$. subcapitata than in M. aeruginosa.

The patterns revealed by the analysis of multidimensional trait diversity indices are evident in the dynamics of the individual fluorescence distributions (Fig. 5; Supplementary Fig. S9-S13). Interestingly, these plots show substantial changes in total fluorescence over time. Observed shifts in fluorescence during the experiment were much more dramatic in $M$. aeruginosa than in $P$. subcapitata, again consistent with our interpretation of the multidimensional indices. The fluorescence distribution dynamic plots also highlight some of the reasons behind changes in trait evenness and richness across light intensities. For example, in M. aeruginosa under low light, broader bands indicate higher richness (it is important to consider the log-scale of the $Y$-axes to correctly evaluate bandwidth), while less variation in colour suggests higher evenness (Fig. 5; Supplementary Fig. S12-S13).

Although we expected increased light limitation-and consequently stronger changes in trait evenness-as a 
Table 1 Results of the linear models with light intensity (midpoint of the range in each light treatment) as predictor of two response variables: TED slope $(=$ slope estimate of each linear fit of TED change over time; Fig. 3) (A), and TED values at carrying capacity $(\mathrm{B})$

\begin{tabular}{|c|c|c|c|c|c|c|c|c|}
\hline & \multicolumn{4}{|c|}{ P. subcapitata } & \multicolumn{4}{|c|}{ M. aeruginosa } \\
\hline & Est. $\left[10^{-5}\right]$ & $\mathrm{SE}\left[10^{-5}\right]$ & $p$-value & $\overline{\text { Adj. } R^{2}}$ & Est. $\left[10^{-5}\right]$ & $\mathrm{SE}\left[10^{-5}\right]$ & $p$-value & Adj. $R^{2}$ \\
\hline \multicolumn{9}{|c|}{ A) TED slope $\sim$ light intensity } \\
\hline Global model & -18.4 & 2.5 & $<0.001$ & 0.75 & -66.4 & 6.1 & $<0.001$ & 0.87 \\
\hline Direct light & -17.7 & 2.2 & 0.001 & 0.93 & -77.7 & 14.7 & 0.006 & 0.84 \\
\hline Indirect light & -12.8 & 2.3 & 0.005 & 0.85 & -64.2 & 9.9 & 0.003 & 0.89 \\
\hline Mixed cultures & -23.6 & 4.6 & 0.007 & 0.84 & -54.9 & 7.1 & 0.001 & 0.92 \\
\hline \multicolumn{9}{|c|}{ B) TED carrying $\sim$ light intensity } \\
\hline Global model & -14.9 & 4.7 & 0.003 & 0.19 & -51.7 & 3.8 & $<0.001$ & 0.52 \\
\hline Direct light & -32.2 & 4.4 & $<0.001$ & 0.78 & -46.3 & 6.5 & $<0.001$ & 0.44 \\
\hline Indirect light & -22.6 & 4.4 & 0.004 & 0.81 & -61.7 & 5.4 & $<0.001$ & 0.71 \\
\hline Mixed cultures & -10.0 & 5.8 & 0.11 & 0.12 & -45.0 & 6.1 & $<0.001$ & 0.49 \\
\hline
\end{tabular}

Regression estimates, standard errors, $p$-values and adjusted $R^{2}$ are indicated. Global models include data points from monocultures (under direct and indirect light) and mixed cultures
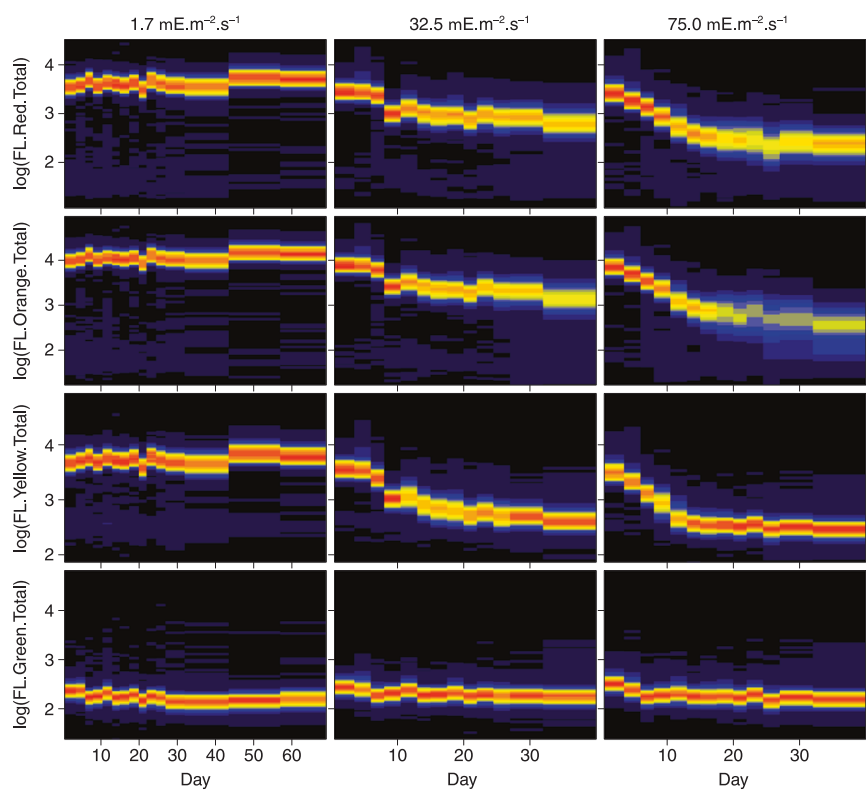

Fig. 5 Fluorescence distribution dynamics in $M$. aeruginosa under direct light. For each time point we computed a binned kernel density estimate using 1000 random cells from each of the two replicates. Note the different $X$-axes, reflecting variation in experimental duration across light treatments. As $Y$-axes are on log-scale, changes in total

consequence of shading by another species, we were unable to detect clear differences between direct light, indirect light and mixed cultures. This might suggest that $P$. subcapitata and $M$. aeruginosa occupy distinct light niches and the patterns we observed were mainly driven by intraspecific competition.

Trait evenness decreased faster and showed a broader range of values in $M$. aeruginosa (Figs. 3, 4) compared to $P$. subcapitata. $M$. aeruginosa also outperformed $P$. subcapitata in terms of growth in the mixed cultures, under all light levels (Supplementary Fig. S1-S6). These
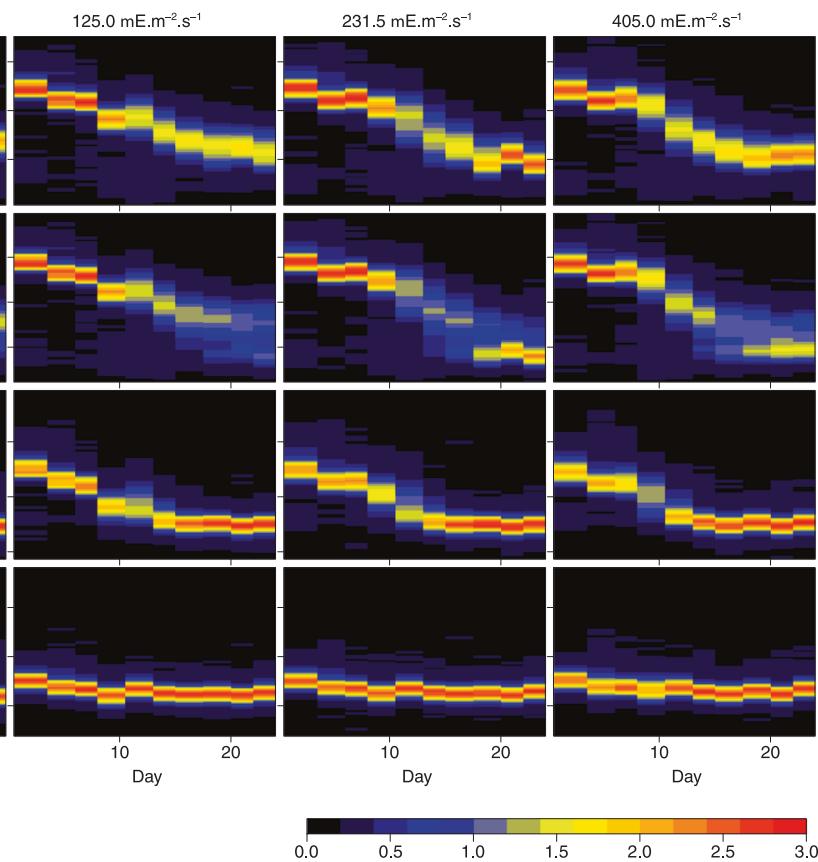

fluorescence are substantial in many cases. Fluorescence reaches a maximum at the lowest light intensity in most channels. The absolute total fluorescence values of different channels are not directly comparable (color figure online)

results suggest that the cyanobacterium is capable of high photosynthetic trait plasticity, as has been previously shown for this group (e.g., [17, 31]). It may also benefit from faster growth rates and a broader array of available pigments compared to the green alga [32, 33]. The importance of phenotypic plasticity (adjustment of pigment ratios to the prevailing light spectrum) in determining the outcome of competition for light has been demonstrated before in phytoplankton, but only at the interspecific level [17]. Most likely in our case, phenotypic changes can be ascribed to plasticity on short time 
scales (days to weeks) after treatments were applied (Fig. 3), while selection on standing genetic variation and evolution (in form of differential reproductive success of genotypes) could have played a role in the patterns towards the end of the experiment [34]. More work will be needed in the future to understand the relative importance of plasticity and evolution in shaping the patterns observed here. Similarly, the generality of our findings across other taxonomic groups, morphological and physiological traits or resource gradients needs to be confirmed by further research.

This study however supports the hypothesis that the regularity in the distribution of individuals along multiple resource acquisition axes increases with decreasing resource availability. Our results reinforce previous findings that resource limitation increases phenotypic heterogeneity in microbes [13], and indicate that a highly restrictive abiotic environment (i.e., low light) does not necessarily select for a reduced trait variability, as often assumed in ecology (the environmental filtering hypothesis) [35]. Instead of convergence towards an optimal phenotype, survival under low resource conditions in our experiment may have induced cellular processes increasing phenotypic variation to find new and beneficial strategies, thereby increasing inter-individual phenotypic distances in trait space. This finding is consistent with the hypothesis that high trait evenness emerges in conditions in which it is advantageous to minimise competition for a limiting resource. On the contrary, when resources are not limiting, organisms may converge towards a limited range of phenotypic profiles that enable fast growth. This argument may also apply to other resources that are available in multiple forms (analogously to light wavelengths), as it is the case for some nutrients (e.g., [13]). In summary, we report novel empirical findings into how resource availability can shape phenotypes in a competitive environment, and demonstrate that conspecific and heterospecific phytoplankton cells tend to differentiate their pigment profile under conditions of low light intensity. Our results represent a step forward in elucidating the mechanisms that maintain coexistence in populations and communities over resource gradients.

\section{Data availability}

The data set analysed during the current study are available in the Zenodo repository at https://doi.org/10.5281/zenodo. 1982920.

Acknowledgements This research is supported by the Swiss National Science Foundation Projects 31003A_144053 and CRSII2_147654. We thank R. Stegmayer for his help in lab work, H. Penson for maintaining algal cultures, D. Fontana for help with figure processing, and M. Kehoe for the inspiring discussions. The Centre for Ocean Life is a Villum Kahn Rasmussen Centre of Excellence funded by the Villum Foundation. We thank the three anonymous Referees for improving the article with their constructive comments. The authors dedicate this work to the memory of M. Stomp and G. Fontana.

Funding This research is supported by the Swiss National Science Foundation Projects 31003A_144053 and CRSII2_147654.

\section{Compliance with ethical standards}

Conflict of interest The authors declare that they have no conflict of interest.

\section{References}

1. Reiss J, Bridle JR, Montoya JM, Woodward G, Montoya M. Emerging horizons in biodiversity and ecosystem functioning research. Trends Ecol Evol. 2009;24:505-14.

2. Clark JS, Bell DM, Hersh MH, Kwit MC, Moran E, Salk C, et al. Individual-scale variation, species-scale differences: Inference needed to understand diversity. Ecol Lett. 2011;14:1273-87.

3. Chesson P. Mechanisms of maintenance of species diversity. Annu Rev Ecol Syst. 2000;31:343-66.

4. Jung V, Violle C, Mondy C, Hoffmann L, Muller S. Intraspecific variability and trait-based community assembly. J Ecol. 2010;98:1134-40.

5. Barabás G, D'Andrea R. The effect of intraspecific variation and heritability on community pattern and robustness. Ecol Lett. 2016;19:977-86.

6. Paine CET, Baraloto C, Chave J, Hérault B. Functional traits of individual trees reveal ecological constraints on community assembly in tropical rain forests. Oikos. 2011;120:720-7.

7. Bolnick DI, Amarasekare P, Araújo MS, Bürger R, Levine JM, Novak M, et al. Why intraspecific trait variation matters in community ecology. Trends Ecol Evol. 2011;26:183-92.

8. Turcotte MM, Levine JM. Phenotypic plasticity and species coexistence. Trends Ecol Evol. 2016;31:803-13.

9. Violle C, Enquist BJ, McGill BJ, Jiang L, Albert CH, Hulshof C, et al. The return of the variance: intraspecific variability in community ecology. Trends Ecol Evol. 2012;27:244-52.

10. Clark JS. Individuals and the variation needed for high species diversity in forest trees. Science. 2010;327:1129-32.

11. De Laender F, Melián CJ, Bindler R, Van den Brink PJ, Daam M, Roussel $\mathrm{H}$, et al. The contribution of intra- and interspecific tolerance variability to biodiversity changes along toxicity gradient. Ecol Lett. 2014;17:72-81.

12. Ackermann M. A functional perspective on phenotypic heterogeneity in microorganisms. Nat Rev Microbiol. 2015;13:497-508.

13. Schreiber F, Littmann S, Lavik G, Escrig S, Meibom A, Kuypers MMM, et al. Phenotypic heterogeneity driven by nutrient limitation promotes growth in fluctuating environments. Nat Microbiol. 2016;1:16055.

14. Zimmermann M, Escrig S, Lavik G, Kuypers MMM, Meibom A, Ackermann M, et al. Substrate and electron donor limitation induce phenotypic heterogeneity in different metabolic activities in a green sulphur bacterium. Environ Microbiol Rep. 2018;10:179-83.

15. Fontana S, Thomas MK, Moldoveanu M, Spaak P, Pomati F. Individual-level trait diversity predicts phytoplankton community properties better than species richness or evenness. ISME J. 2018;12:356-66.

16. Stomp M, Huisman J, De Jongh F, Veraart AJ, Gerla D, Rijkeboer $\mathrm{M}$, et al. Adaptive divergence in pigment composition promotes phytoplankton biodiversity. Nature. 2004;432:104-7.

17. Stomp M, van Dijk MA, van Overzee HMJ, Wortel MT, Sigon CAM, Egas M, et al. The timescale of phenotypic plasticity and its 
impact on competition in fluctuating environments. Am Nat. 2008;172:E169-85.

18. Reynolds CS. Ecology of phytoplankton. Cambridge: Cambridge University Press; 2006.

19. R-Core-Team. R: a language and environment for statistical computing. Vienna, Austria: R Foundation for Statistical Computing; 2013.

20. Fontana S, Jokela J, Pomati F. Opportunities and challenges in deriving phytoplankton diversity measures from individual traitbased data obtained by scanning flow-cytometry. Front Microbiol. 2014;5:1-12.

21. Pomati F, Kraft NJB, Posch T, Eugster B, Jokela J, Ibelings BW. Individual cell based traits obtained by scanning flow-cytometry show selection by biotic and abiotic environmental factors during a phytoplankton spring bloom. PLoS One. 2013;8:e71677.

22. Dubelaar GBJ, Geerders PJF, Jonker RR. High frequency monitoring reveals phytoplankton dynamics. J Environ Monit. 2004;6:946-52.

23. Liaw A, Wiener M. Classification and regression by random orest. R News. 2002;2:18-22.

24. Breiman L. Random forests. Mach Learn. 2001;45:5-32.

25. Thomas MK, Fontana S, Reyes M, Pomati F. Quantifying cell densities and biovolumes of phytoplankton communities and functional groups using scanning flow cytometry, machine learning and unsupervised clustering. PLoS One. 2018;13:e0196225.

26. Fontana S, Petchey OL, Pomati F. Individual-level trait diversity concepts and indices to comprehensively describe community change in multidimensional trait space. Funct Ecol. 2016;30:808-18.
27. Stomp M, Huisman J, Stal LJ, Matthijs HCP. Colorful niches of phototrophic microorganisms shaped by vibrations of the water molecule. ISME J. 2007;1:271-82.

28. Mouillot D, Graham NAJ, Villéger S, Mason NWH, Bellwood DR. A functional approach reveals community responses to disturbances. Trends Ecol Evol. 2013;28:167-77.

29. Kraft NJB, Valencia R, Ackerly DD. Functional traits and nichebased tree community assembly in an Amazonian forest. Science. 2008;322:580-2.

30. Weiher E, Keddy PA. Assembly rules, null models, and trait dispersion: new questions from old patterns. Oikos. 1995;74: 159-64.

31. Tandeau de Marsac N. Chromatic adaptation by cyanobacteria. In: Bogorad L, Vasil IK, eds. The photosynthetic apparatus: molecular biology and operation. San Diego, CA: Academic Press; 1991. p. 417-44.

32. Glazer AN. Phycobilisome a macromolecular complex optimized for light energy transfer. Biochim Biophys Acta. 1984;768: $29-51$.

33. Richardson K, Beardall J, Raven JA. Adaption of unicellular algae to irradiance: an analysis of strategies. New Phytol. 1983; 93:157-91.

34. Ellner SP, Geber MA, Hairston NG. Does rapid evolution matter? Measuring the rate of contemporary evolution and its impacts on ecological dynamics. Ecol Lett. 2011;14:603-14.

35. Kraft NJB, Adler PB, Godoy O, James EC, Fuller S, Levine JM. Community assembly, coexistence and the environmental filtering metaphor. Funct Ecol. 2015;29:592-9. 